\title{
A Theoretical Overview of Bioresponse to Magnetic Fields on the Earth's Surface
}

\author{
Pietro Volpe ${ }^{1,2}$ \\ ${ }^{1}$ Department of Biology of the "Tor Vergata" University of Rome, Rome, Italy \\ ${ }^{2}$ National Institute of Biostructures and Biosystems, Rome, Italy \\ Email: volpe@uniroma2.it
}

Received 8 July 2014; revised 3 August 2014; accepted 25 August 2014

Copyright (C) 2014 by author and Scientific Research Publishing Inc.

This work is licensed under the Creative Commons Attribution International License (CC BY). http://creativecommons.org/licenses/by/4.0/

(c) (i) Open Access

\section{Abstract}

This survey points to the mechanisms of bioresponse caused by magnetic fields (MFs), paying attention to their action not only on ions, molecules and macromolecules, but also on cells, tissues and organisms. The significance of findings concerning the MF-dependence of cell proliferation, necrosis or apoptosis was judged by comparing the results obtained in a solenoid, where an MF can be added to the geomagnetic field (GMF), with those obtained in a magnetically shielded room, where the MFs can be attenuated or null. This comparative criterion was particularly appropriate when the differences detectable between the data provided by experimental samples and the data provided by control samples were rather small, as observed in estimating the MF-influence on total DNA replication, RNA transcription and polypeptide translation. The MF-induced inhibition of apoptosis was considered as a risk potentially leading to accumulation of cancer cells. The analysis also surveyed the MF-dependence of the interactions between host animal cells and infecting bacteria. In relation to studies on the origin and adaptation of life on the Earth, theoretical insights paving the way to elucidating the MF-interactions with biostructures and biosystems of different orders of organization evaluated the possible involvement of the so-called "biological windows". Analogously to what is known for ionizing radiations, the efficiency of the applied MFs appeared to depend on the complexity of their biological targets.

\section{Keywords}

Magnetic Fields vs. Living Matter, Biological Windows, Biodysfunctions, Apoptosis vs. Neoplasia

\section{Introduction}

The modern knowledge of the origin of the GMF and its reversals emerged from an intriguing controversy. A superficial look at the change of the dipole moment over a century, rejecting the correct idea that the Earth's MF 
had reversed polarity [1], erroneously led to believing that the GMF has decayed, since creation, no more than ten thousand years ago [2]. However, the subsequent analysis_-showing that reversals of the main field do take place [3] — was supported by studies on geomagnetism and paleomagnetism that correlated the rock magnetism with the history and origin of the Earth's MF, whilst an effort was made to determine the magnetic structures of minerals and their stability as a function of time [4]. To understand how the GMF was generated, this investigation provided equations describing electromagnetic and thermal conditions in the Earth's core. Thus, the lithosphere magnetic anomalies were associated with magnetized rocks, such as ore concentrations, magmatic and ore bodies [5] [6].

A series of old and new contributions revealed that the Earth's surface is protected by an MF-mantle constituted of several components. The Sun, with its electromagnetic and corpuscular irradiations, contributes to forming a complex MF on the Earth, while the solar wind (SW), rotating with the Sun's rotation around its axis, fills our celestial space [7]. The SW roughly consists of four sectors: the first two, showing magnetic force-lines directed away from the Sun, are indicated as positive; the other two, showing opposing force-lines, are indicated as negative [8].

Influence of the solar activity on the GMF was observed at the beginning of the XXth century, but experimental data were collected later. It was seen that a magnetic storm (MS) is generated by the plasma flows from the regions of bursts of solar activity [4]. They arise ten or more times in a month, with an average frequency of about eleven years, corresponding to the oscillations in the same solar activity. The MS reaches the Earth two days after the Sun's bursts. With respect to the GMF, an MS was subdivided into four phases, defined as preliminary, initial, main, and recovery. During the preliminary phase, small changes in the GMF were detected. During the initial phase, a drastic variation in the GMF occurred. During the main phase, quasi-periodical oscillations-low-frequency MFs, extremely low-frequency (ELF) MFs, and a drop in the horizontal component of the GMF - took place. During the recovery phase, all these variations faded away.

It was verified that the flow of protons, rotating around the Earth under the action of the GMF, forms a ring defined as equatorial current, whose MF attenuates the GMF in the main phase of an MS. At the same time, the intensity of the GMF - varying from about 35 microT at the equator (where it is almost parallel to the terrestrial surface) to about 70 microT at the Earth's magnetic poles (where it is almost vertical) - is perturbed by such an MS. The MS could produce alterations of the GMF lasting from several hours to several days all around the Earth, with a maximal magnitude of about 5 microT at the high latitudes and about 1 microT in the mid-latitudes.

In addition to the 11-year periods of magnetic fluctuations due to the Sun's activity, it was measured that there are other periods of variation in the GMF: an 1-day cycle due to the Earth's rotation; a 6 - 7-day cycle corresponding to one sector of the SW; a 13 - 14-day cycle due to the passage of two sectors of the SW; a 27-day cycle corresponding to the period of the Sun's rotation around its own axis [4]. Crucial rhythms, also correlated with these cycles, were listed with accuracy by Zhadin [9]: a 29.5-day cycle corresponding to the Moon's synodic period; an 1-year cycle corresponding to the period of the Earth's revolution; some cycles of the solar activity which were not so prominent when compared to the main 11-year cycle (2-, 3-, 8-, 22-, and 35-year cycles). To consider besides the continuous spectrum of quasi-periodic, low-frequency processes in the GMF (with maxima near $100,21,8,3,1,0.1,0.01$, and $0.001 \mathrm{~Hz}$ ) which are caused by the oscillations in the plasmasphere and the magnetosphere of the SW and by the Schumann resonances in the ionosphere of the Earth [7]-[11].

\section{Perturbations of Earth's MF-Assemblage Caused a Number of Biodysfunctions}

Despite the obvious difficulty to detect the specific biological effect exerted by each one of the components of the MF-mosaic formed around the Earth, valuable results were gathered by analyzing the bioresponse to the GMF taken as a whole.

Prokaryotes. The attenuation of the GMF in a magnetically shielded room with shielding indexes of several tens up to 100 induced a decrease of bacterial vital functions [12].

Plants. It was shown that the GMF plays a role, on the one hand, in total plant branching, in orientation of the root branches, in metabolism and proliferation of the root meristem cells and, on the other, in protein synthesis occurring in several vegetal cells [13]-[16]. GMF variations caused either changes of the mitochondrial structure in meristem of seedling roots of pea, flax, and lentil [14] or a $68 \%-75 \%$ reduction of cell proliferation accompanied by a variation in RNA and protein biosynthesis [15]. 
Animals. A change in timing of fibroblast division, an increase in sensitivity of several cell cultures to poisons, and a decrease in erythrocyte sedimentation were correlated with GMF anomalies [17] [18]. When guinea pigs were subjected for 30 min to a GMF ten times weaker, in their blood there occurred an increase of the epinephrine and histamine levels vs. a decrease of the serotonin concentration [19]. In rats, the hypogeomagnetic conditions caused a decrease of learning and an increase of hippocampus catecholamine [20]. The orientation of sea gulls or pigeons was strongly GMF-dependent [21]-[24]. In the acoustico-lateral area of the skate's brain there were two groups of neurons, one excited by the MF with the south sense and another inhibited by the MF with the north sense [25].

\section{In Animals There Appeared Heavy Dysfunctions Correlated with an MS}

Notwithstanding the difficulty of detecting biological dysfunctions caused by specific components of the Earth's MF-mosaic, it was observed that given bioresponses were clearly MS-dependent. In fact, the MS was correlated with anxiety and irritability and with lower attention and accuracy on the job: these symptoms caused an increase of the errors made by pilots and an increment of the road accidents [26]. Under an MS, the glaucoma attacks increased [27], and the acute cardiovascular diseases became more frequent [28]. During the days of GMF perturbation by an MS, variations were observed in conditioning the activity of rats, pigeons and dogs, and reactions of single neurons were registered in the motor neocortex of cats [29]. Under the exceptional double planetary MS of 1984, specific parameters of the cardiovascular system and ultrastructure of cardiomyocytes were checked in rabbits: at the initial and main phases of the storm, the normal circadian fashion in each cardiovascular parameter was lost; desynchronization increased with the storm, and an abrupt drop of cardiac activity was detected; the main phase of the storm was accompanied by a degradation of mitochondria in cardiomyocytes [30]. Cell cultures, coming from mouse, hamster and trout, were employed to show that morphological and functional states are associated with GMF changes. The MS-disturbance of the GMF caused stepwise changes in the properties of cell surface, appearance of heterokaryons, and strengthening of cell-to-cell adhesion and aggregation [31]. Lastly, there was an effect of 0.25 T DC MFs on microcirculation in rabbits [32], while the convergence of artificial DC MFs and GMF produced a harmful impact on cardiovascular regulation [33].

\section{An Interdisciplinary Debate Was Focused on the Mechanisms of Bioresponse to MFs}

The data reported above, showing examples of bioanswer to the MF-system formed on the Earth's surface, stimulated a discussion on the mechanisms that could be on the basis of the interaction between MFs and living matter [34]. A first task, pointing to a possible involvement in MF-bioeffects of resonance at the cyclotron frequency under combined influence of DC and AC MFs [35], was followed by an assumption basing these bioeffects on an ion parametric resonance model. It was suggested that the rise of a ponderomotive force caused in a biostructure by non-uniform DC MFs could explain the magnetotropism of plants (where the "paramagnetic cells", representing a minor biomass, would be forced towards a point of maximal field); instead, the "diamagnetic cells", representing a major biomass, would be forced towards the opposite direction [36].

Analyzing the interaction of the ionic channels in neuromembranes with the spreading action potential, it was proposed that "external" MFs should interact with hypothetical "internal" MFs created by the ionic channels and nodes of Ranvier [37]. With reference to non-equilibrium systems in low-frequency/low-intensity MFs, a distinction was made between their direct perception by biomolecular structures and their signalling role through the central nervous system [38]. Of interest was the influence of GMF variations on the agglutination reactions and on the Piccardi test [39]. In an attempt to explain the biovariations caused by GMF perturbations, the occurrence of dissipative clathrate structures in water and non-equilibrium conditions was suggested [40].

Zhvirblis paid attention to the correlation of several medico-biological tests with the index of GMF perturbation in which sector boundaries of the interplanetary MF, associated with the SW, were taken as zero [41]. His analysis revealed that the direction along the phase trajectory contour changed when the Earth crossed the sector boundary (due to sensitivity of the Piccardi test to polarity of a radial component of the interplanetary MF, it was assumed that this sensitivity could be caused by the geomagnetic micropulsations in the range from 0.007 to $0.1 \mathrm{~Hz})$.

Other investigations regarded the influence of weak (non-thermal) and ultraweak (natural) AC MFs on inorganic and biological systems. It was proposed that the calcium-binding proteins constitute the place of primary 
action of combined MFs. These proteins would cause a splitting of energetic levels of the calcium ion oscillation in the protein (Zeeman effect). Being the width of the splitting equivalent to the cyclotron frequency of calcium and the applied DC MF, the parametric resonance was thought as a mechanism to increase ionic energy. The DC, AC and DC + AC MFs could change the polarization degree. So, the biological effects of MFs could be induced by the same polarization degree of the calcium ion oscillation [42] [43].

To define an idealized quantum model, an investigation pointed to the mechanism of magnetosensitive ion binding by proteins under the influence of external AC MFs [44]. It turned out that the dissociation probability of an ion-protein complex depended on frequency and amplitude. This was shown to be a consequence of the interference of angular modes of the ion wave function [45].

In relation with the orientation of ions, molecules and cells in a DC MF [46], attention also was drawn on the problem of the dielectric response of tissues [47] and on the thermal oscillations of the calcium ion in a calcium-binding protein under the influence of DC + AC MFs. It emerged that the DC MF caused the Larmor precession of ion oscillations [48]. Since the cyclotron frequency is twice the Larmor frequency, this event suggested that the phenomenon could serve as a basis for increasing the kinetic energy of the calcium ion due to parametric resonance. In agreement with this idea, an AC MF perpendicular to a DC MF (or an AC MF parallel to a DC MF) was expected to increase the ionic energy, and a maximal effect was expected at the cyclotron frequency of an AC MF.

The question of the ion motion in a macromolecule generated an attractive discussion. Zhadin [49] tried to solve equations of the ion motion in a macromolecule under influence of the MFs, considering the damping effects and the influence of particles surrounding this ion. In contrast with Blanchard and Blackman [36], his study showed that the possibility of parametric resonance should not be credible for the AC MF frequencies, being many orders of magnitude lower than the natural frequency of an ion in a macromolecule. The expectation was that the MFs would cause changes in energy of the ionic thermal motion: this would be sufficient to trigger variations in the conformational state of a macromolecule. Binghi [50] problematically judged these argumentations [49] [51]: for him, the statement that the interaction of weak MFs with ions trapped in protein cavities could not produce detectable biological effects through changing the character of the ion orbits [52] was sub judice.

With some bearing on this, two divergent orders of calculations should be taken into consideration.

First, it was assumed that weak ELF MFs would affect intracellular DNA directly, because the MFs should exert a force of the order of $I_{\mathrm{DNA}}=1.6 \times 10^{-13} \mathrm{~A}$ on the currents that flow in the double stranded macromolecule [53].

Second, it was claimed that this conclusion would not be in agreement with the physical rules, because the force on the current carrying strands generated, for example, by an MF, $B=5$ microT, should be extremely weak [54], i.e., equal to $F=B I_{\mathrm{DNA}}=8 \times 10^{-19} \mathrm{~N} / \mathrm{m}$. In other words, a motion of a $1 \mathrm{~mm}$ long strand through a distance equal to that of the strand diameter of $2 \mathrm{~nm}$ would represent the energy of $1.6 \times 10^{-33} \mathrm{~J}$ or $3.7 \times 10^{-13} \mathrm{kT}$. Thus, the energy transfer from the MF on the strand should be negligible.

The investigations performed on neurons exhibited a special importance for studies on the mechanisms of bioresponse to MFs. Del Moral and coworkers integrated the Hodgkin and Huxley equation for the bioelectric impulse by considering the membrane as a Kirchoff electric knot [55]. Integration was performed under applied AC MFs by partitioning the bioelectric impulse in its de-polarisation (D) and re-polarisation (R) plus hyper-polarisation $(\mathrm{H})$ regimes. In the cases of spontaneous regimes, there resulted time variations of the $\mathrm{D}$ and $\mathrm{R}$ $+\mathrm{H}$ voltages in reasonable agreement with experiments on single unit neurons of Helix aspersa. Besides, the same authors suggested that the window effect [56] should be a consequence of the phospholipid superdiamagnetism and $\mathrm{Ca}^{2+}$ coulomb explosion in the outer surface of the neurons (the effect should be a resonance of Lorentzian shape when the MF frequency matches the neuron spontaneous frequency).

Some attention also should be paid to the so-called water hypothesis. It assumed that the aqueous medium, outside and inside the cell, is the actual target through which the biological effects of ELF MFs would take place [57] [58]. This assumption was based on the idea that the water dissociation products would serve as "second messengers" able to modulate the intracellular metabolism [59] [60]. Actually, the modulation of cell hydration was thought to be the mechanism whereby extremely low concentrations of biological active substances and weak physical signals, MFs for instance, would exert significant biological effects [61]. Most of these stimuli should be unable to activate ionic channels and receptors in a membrane, but they would function to change the cell volume leading, consequently, to a number of dysfunctions [62] [63].

A further hypothesis emerged from Molecular Microwave Spin Chemistry [64]. It tried to explain the 
MF-dependence of a total body physiological function [65]. Both components of the electromagnetic field, electric and magnetic, should have effects, since the former (efficiently absorbed by water) would interact with the electric dipoles of biosystems (molecules, ions, membranes), while the latter would interact with the magnetic dipoles of electron spins whose carriers are paramagnetic molecules $\left(\mathrm{O}_{2}\right)$, metal ions, radicals and ion-radicals. The individual radicals should be unable to cause either any magnetic or electro-magnetic biological effects. These could rise only when pairs of radicals or ion-radicals are present. The spin states of such pairs- "singlet" (with zero total electron spin) or "triplet" (with unit spin)—would differ in their reactivity, displaying high spin selectivity (a microwave field would induce spin triplet-singlet transitions, changing the spin state and reactivity).

The analysis of this chain of events led to discover that, since the magnetic isotope ${ }^{25} \mathrm{Mg}$, for instance, could become a spin catalyst of enzyme phosphorylation reactions, their kinetics would depend on the nuclear magnetic moment and on both the magnetic and microwave fields [66].

The enzymatic reactions of ATP synthesis should be spin-selective, whilst the spin conversion and chemical activity of ion-radical pairs would depend on the nuclear magnetic moment, external MF, and microwave excitation.

This framework implied that, when mitochondria are immobile or slowly rotate with a frequency below $1 \mathrm{~Hz}$, the anisotropic component of superfine interaction (at ${ }^{31} \mathrm{P}$ or ${ }^{25} \mathrm{Mg}$ nuclei in ion-radical pairs) should not be averaged to zero. Meanwhile, the spin conversion rate of the pair should depend on the orientation of its molecular axes particularly relative to the terrestrial MF.

These hypotheses shed light on various physico-chemical items, but no theories were sufficiently worked up to correlate the bioreactivity to the complex MF-world of the Earth's surface in a unified fashion, taking into consideration the different levels of organization of the living machinery: from atoms, molecules and macromolecules to cells, tissues and entire organisms. Buchachenko and Berdinsky [66] made only an attempt in this direction, when they proposed that the ATP synthesis-due to its intrinsic MF-dependence-presumably would induce a chain of reactions able to control, in turn, the orientation of birds and fishes during their seasonal migrations.

\section{A Correlation Seemed to Exist between Biocomplexity and Sensitivity to an MF}

To complement the debate focused on the theoretical aspect of the MF-action on biological targets, it was worth evaluating their MF-sensitivity comparing the behavior of biostructures of relatively lower complexity (molecules, macromolecules) with that of biostructures of relatively middle (cells in a given cell-cycle phase, proliferating cell cultures) and higher (apoptotic cells, eukaryotic cells infected with bacteria, embryos) complexities. There exist two sources of MFs to which bacteria, plants and animals, independently on the order of their complexity, are subdued: the MF-mantle on the Earth's surface essentially was formed by a natural zero frequency MF, i.e. GMF; in the XXth century, industrialization "enriched" this mantle with both artificial DC and AC MFs of various intensities. On the basis of this observation, a question concerning the adaptation of organisms to these new unexpected fields arose: would a cell maintain its normal functions in an MF-environment characterized by an intensity much greater than that in which it naturally differentiated? This question accompanied that pointing to the level of biocomplexity. The struggle for existence- under pressure of any "new" component of the Earth’s MF-mosaic - correspondingly could favour the development of given parts of an organism.

The information on all this was contradictory: the negative effects of ELF MFs, generated by $50 \mathrm{~Hz}$ high voltage power lines, video screens, electric blankets and other home appliances, were forcibly denounced as time went by [67], but vice versa a benefit from the low-energy pulsed MFs in non-union bone fracture healing and in cell regeneration was found [68]. Divergences also regarded the fact that the MF-dependence of a number of biological structures and functions was due not only to peculiarities of given laboratory facilities, or methodological accuracy, but also to feasibility of the programmed experiments.

\subsection{Biotargets of Lower Complexity Exhibited a Relatively Higher MF-Stability}

At the molecular level, a role of the calcium ions in transduction of the MF-influence on cell proliferation was described [69], and a weak modulation of the calcium traffic through a membrane by an MF at $60 \mathrm{~Hz}$ was reported [70]. Factors distorting cytosolic calcium measurements in Jurkat cells, during their exposure to ELF MFs, were revealed, and a spiking of calcium ions in osteosarcoma cells, exposed to ELF MFs, was detected 
[71]. Small effects of the exposure to ELF MFs on acetylcholine release in hippocampus of freely moving rats or membrane-bound enzymes also were documented [72].

At the macromolecular level, some AC MF-induced DNA damage in human blood cells was observed [73], and a specific cytogenetic effect of $900 \mathrm{MHz}$ microwaves on human lymphocytes was seen [74]. There were increments in X-ray-induced gene mutations by exposure to AC MFs in NF-kappa B-inhibited cells and in hypoxanthine-guanine phosphoribosyl transferase gene mutations by exposure to electric fields [75]. There was a weak influence of ELF MFs on both in vitro DNA and RNA polymerase activity and cell-free mismatch repair [76]. An effect of UVB radiation and MFs at $100 \mathrm{~Hz}$ on DNA synthesis in Jurkat cells was detected [77]. An ELF MF decreased adenylate kinase activity in retinal rod outer segment membranes, and an electric "relaxation" was shown to occur in lipid-bilayers after exposure to weak magnetic pulses [78]. A relative MF-stability of DNA replication [79] and a moderate MF-sensitivity of RNA transcription and translation [80] were found in friend cells.

\subsection{Biotargets of Middle Complexity Exhibited a Moderate MF-Stability}

At the cellular level, an MF-dependence of the membrane state was established through cytofluorimetry [81]. ELF MFs, as well as heat shock, proved to increase microvescicle motility in astrocytes [82]. Both sedimentation and aggregation of erythrocytes were MF-dependent [83]. At the level of cell-cycle kinetics and at that of cell culture proliferation, there was an agreement regarding the MF-effect: variations of Friend erythroleukemia cell proliferation, following the addition or deprivation of MFs [84], were consistent either with alteration of the onset of S-phase established after a $50 \mathrm{~Hz}$ MF exposure of normal human fibroblasts [85] or with change of the growth and colony forming ability after exposure of budding yeast to $50 \mathrm{~Hz}$ MFs [86].

\subsection{Biotargets of Higher Complexity Exhibited a Relatively Lower MF-Stability}

The examples of biological systems characterized by various orders of higher complexity abounded. The interaction between prokaryotic and eukaryotic cells was one of them: Figure 1 showed that, under normal conditions of the GMF, a single Mycobacterium tuberculosis (MTB) entered a single human macrophage (HM), whilst an extremely large HM phagolysosome rapidly digested it; under a moderately increased DC MF, about ten MTBs entered a single HM, while smaller HM phagolysosomes became unable to digest them; at the end of the process, the same HM died and the infecting mycobacteria multiplied [87] [88].

The question about the MF-dependence of the "programmed cell death" concerned a further example of MFsensitivity by a biotarget of higher complexity: there was a repercussion of the exposure to MFs on the calcium channel currents using the patch clamp technique [89]; a membrane dielectric change accounted for induction of apoptosis in human promyelocytic leukaemia cells [90]. In terms of correlation between ion homeostasis and apoptosis, similarly to Annexin $\mathrm{V}$ counteracting apoptosis by inducing calcium influx in human lymphocytic $\mathrm{T}$ cells [91], MF caused an apoptosis inhibition via modulation of $\mathrm{Ca}^{2+}$ influx [92].

The embryological level was considered per antonomasia as an example of MF-sensitivity by biotargets of higher complexity: an effect of $10^{-6}$ to $10^{-5}$ T MFs on chicken embryogenesis was found [93]; a continuous irradiation with 200 microT at $50 \mathrm{~Hz}$ caused a striking 6-day retard in the tadpole metamorphosis [94]; an MFeffect on wing size parameters was observed in Drosophila melanogaster [95].

These results were not always devoid of contradictions, of course. Anyway, the sensitivity to MFs appeared to be a general property of biostructures and biosystems, at all levels of their organization: the required MF-intensity tended to be relatively higher when inducing a visible change in a "small" biotarget (in a molecule or in a macromolecule) and relatively lower when inducing a visible change in a "large" biotarget (in a system of cells or in an embryo).

\section{An MF-Induced Decrease of Apoptosis Led to an Increase of Surviving Cancer Cells}

The hypothesis, suggesting that the rescue of damaged cells may be the mechanism explaining why MFs, which are not mutagenic per se, are often able to increase mutation and tumour frequencies [92], had a particular resonance, because it implied an interconnection of all biostructural levels. But what should one say about the framework that allowed the discovery of such an important issue? 


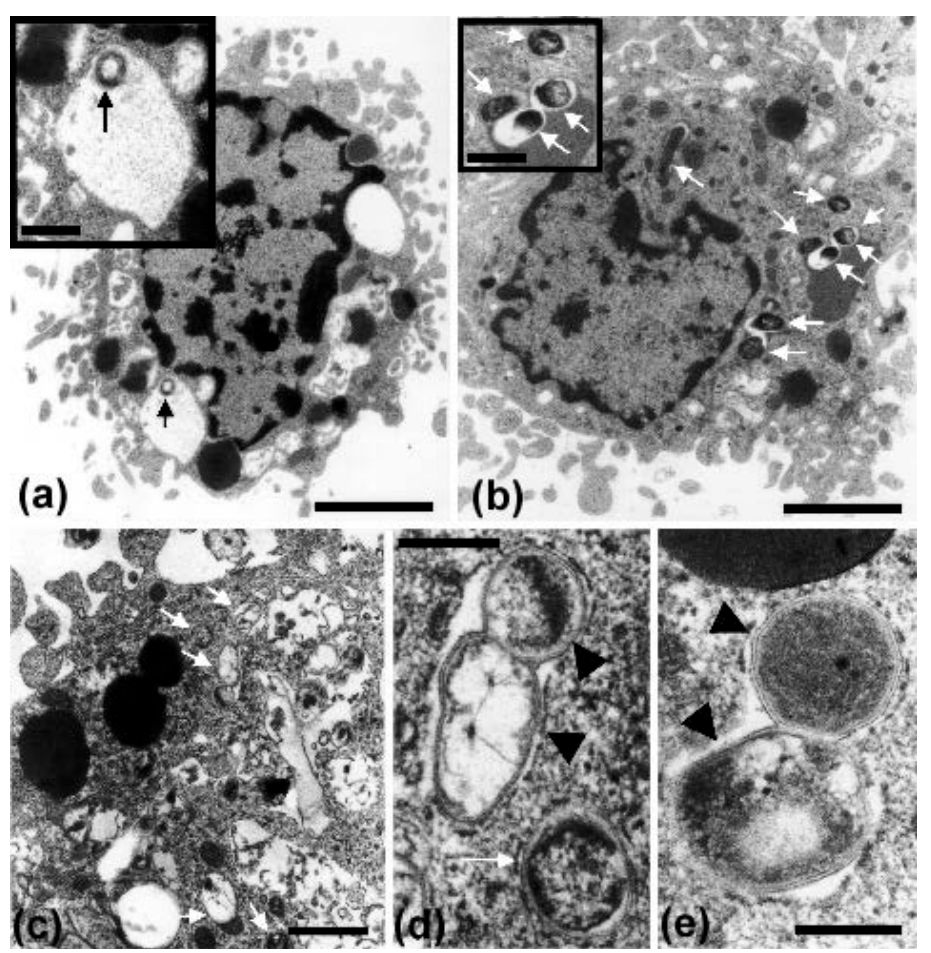

Figure 1. Zero-frequency magnetic fields induced morphological changes in human macrophages infected with Mycobacterium tuberculosis [87] [88]. (a) In a control, unexposed macrophage, one mycobacterium (dark arrow) was located inside a cytoplasm vacuole, as magnified in the upper left corner; (b) In an experimental macrophage exposed to $91 \mathrm{mT}$, several mycobacteria (white arrows) were located inside smaller cytoplasm vacuoles that, in some cases, appeared to be fused with each other; (c) In an experimental macrophage exposed to $670 \mathrm{mT}$, several mycobacteria (white arrows) were essentially free in a cytoplasm unable to form digesting vacuoles; (d) (e) Under $670 \mathrm{mT}$, the free mycobacteria divided, yielding daughters (dark arrowheads) characterized by proper shape and chromatin condensation. The bars measure 2.5 micrometers in (a) and (b), 1.0 micrometer in (c), and $300 \mathrm{~nm}$ in (d) and (e). In the inserts to (a) and (b) they measure $500 \mathrm{~nm}$.

As reviewed by Markov [96], permanent magnets were successfully used for therapeutic purposes, since they offered an easy tool to directly treat the site of injury, the source of pain, or the locus of inflammation. At variance, independently on the type of field, DC or AC, MF-intensity (from $10^{-7}$ to $10 \mathrm{~T}$ ), or living architecture (from in vitro cells to organisms), the research did account for a link between MFs and tumorigenicity [97]: MFs increased tumour cell survival after various cytosidal therapies [98]; MFs were claimed to enhance the mutation rate of cells treated with mutagens [99] [100]; there was an MF-dependent increase of the tumour rate in cancer-prone mice strains [74]. Nonentheless, no direct tumorigenic or mutagenic effects were attributed to MFs [101] [102], as supported by the fact that no DNA damages were detected after exposure to MFs [73] [103]. The MFs rather inhibited the cancer cell metabolism, lowered its malignancy and restrained its rapid growth. The properties of the apoptotic cancer cells significantly changed under an MF action: heterochromatin highly condensed and coagulated with the internal nuclear membrane; endoplasmic reticulum expanded and fused with the external cellular membrane; numerous apoptotic bodies, packed by the cellular membrane, were devoured by lymphocytes [104].

In spite of this achievement, a mechanism that tentatively could explain the interaction between MFs and cancer was not proposed. An idea about such a mechanism emerged, instead, when a multidisciplinary work demonstrated that DC MFs-from about $1 \mathrm{mT}$ to about $100 \mathrm{mT}$-decreased in an intensity-dependent fashion the extent of cell death by apoptosis [87] [92] [105]. This decrease (like in Figure 2(c)) did not appear to be due 
to a variation in the mode of cell death, to necrosis, for instance: the presence of MFs rather allowed an indefinite survival and reproduction of the cells hit by apoptogenic substances. The "protective" influence was mediated by the ability of the fields to enhance $\mathrm{Ca}^{2+}$ influx from the extracellular surrounding [92]. The phenomenon was limited to cell species where the influx of $\mathrm{Ca}^{2+}$ ions had an antiapoptotic action. It led one to assume that MFs, by altering/restoring the equilibrium between cell death and cell proliferation, should be able to increase a tumour mass because of a parallel decrease in it of apoptotic cells [88]. This generalization stimulated new studies by virtue of the alarm that a correlation between tumour frequency and exposure to MFs could mean for future evolution of living matter on the Earth. The alarm was reinforced, first, by the confirmation that, after two, four or six days of exposure to an MF, a gradual decrease in apoptosis vs. a gradual increase in cells with a necrotic morphology were described [106] and, second, by pertinent observations: despite an ELF pulsed-gradient MF inhibited murine malignant tumour growth [104], ELF MF radiation induced cancerogenesis particularly in the case of leukaemia in whose development a role had to be assigned to $\mathrm{Ca}^{2+}$ ion transport and apoptosis [107]; under MFs at densities from 10 to $15 \mathrm{~T}$, cell cytoskeleton and cell differentiation heavily changed [108]. In any case, if the paradoxical ability of MFs to facilitate the growth of a neoplastic mass-without being mutagenic per se-might signify, in a clinical language, that the interaction of MFs with living matter should deal with an escape from a normal cell differentiation leading to cancerogenesis [88], the question about a possible risk to Mankind, caused by long-term exposure to weak MFs, would become, in general, a concern in that even small MF-effects could have profound public health implications [109].

\section{Conclusions}

Even if the observations considered by this overview showed a generalized influence of MFs on biological targets, the analysis of the data available on a larger scale paved the way towards the assumption of an inverse correlation between the degree of sensitivity to MFs and the order of complexity of these targets [110]. The assumption was judged in relation to the inverse correlation established several years ago between the intensity of ionizing radiation and the position of species in evolution: the highest tendency to radiostability was found to characterize bacteria; the lowest tendency to radiostability was found to characterize mammals [111]. In this respect, some attention should be paid to the linkage between two phenomena: biological windows [56] were observed in the curve that reflected the development of myosin phosphorylation against the MF flux density measured in microT [112]; the target theory suggested that even extremely weak ionizing radiations should lead the cell to die [113]. This issue was expected from the fact that in experimental conditions the function, describing the development of cell survival vs. the intensity of ionizing radiation $f=e^{-D / D 1}\left[1-\left(1-e^{-D / D 2}\right)\right]^{N}$, where $D$ is the dose, $D 1$ is the single shot, $D 2$ is the total split dose, and $N$ is a target-hit extrapolation number, never parallels the abscissa, in Gy, starting from its initial shoulder [114].

Therefore, the variation of sensitivity to MFs as a function of biological complexity presumably might depend on the amount of biological windows present in a given irradiated biostructure. In the case that the number of these windows should be very large-let us consider the cycling Friend erythroleukemia cells [84] or the developing tadpoles [94] as examples of targets with notable dimensions-the sensitivity to MFs would be highly significant. In the case that the number of these windows should be very small or below a needed threshold-let us consider single molecular reactions as examples of targets with minimal dimensions [79] [80] — the sensitivity to MFs would become stochastic or absent. From this angle of view, an intensive metabolism would keep "opening" all biological windows of a biostructure, but a weak metabolism should reduce their number or "close" them at all. The behaviour of cell viability or cell necrosis as a function of the MF-intensity might account for such an issue fairly well: the MTB infection, leading a HM to its internal dissolvement, attenuated at the same time its sensitivity to MFs (Figure 2); similarly, a partial attenuation of MF-sensitivity was exhibited by the apoptotic patterns of MTB-infected HMs [87] [88] [105] [110] and by their monoclonal antibody reactions, shown by expression of the CD14 and CD64 membrane protein markers (Figure 3).

Summarizing about the survival, for at least three and half billions of years, of thousands and thousands of prokaryotic and eukaryotic species in the natural MF-mosaic of the Earth's surface, any reasonable prediction on their future evolution-following possible MF "subtractions" (as in the extraterrestrial space) or MF "additions" (as in the electrified areas) [110]—should be questionable. Will the artificial MF-variations, especially when prolonged, cause differential dysfunctions to biosystems, by virtue of their complexity? Will this danger regard first of all the higher animals, the humans, as in the case of the ionizing radiation, since their body comparatively should be full of "opening" biological windows? In a survey mainly devoted to geophysicists, biochemists, 
molecular biologists and physicians, it seemed not out of place to stress that these anxious interrogatives deserve further experimental investigations necessarily from positions of pure science.

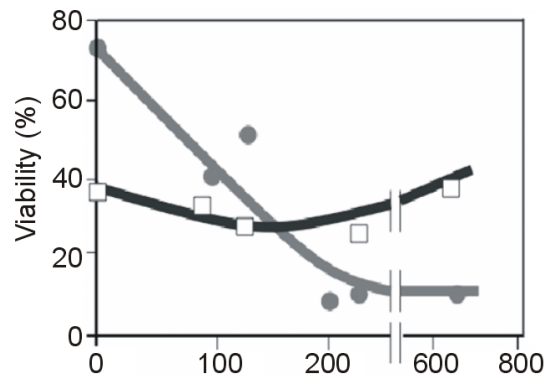

(a)

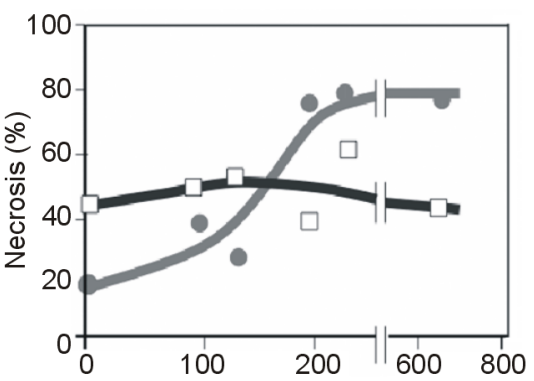

(b)

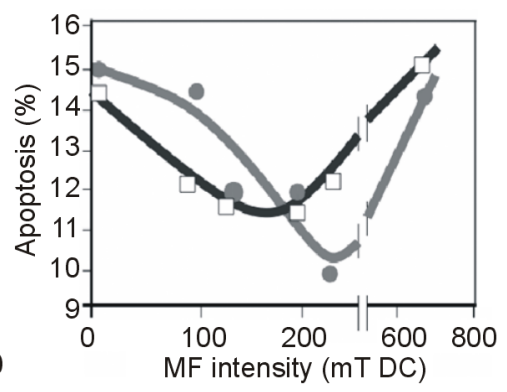

(c)

Figure 2. In human macrophages the infection with Mycobacterium tuberculosis attenuated the MF-dependent variations of viability, necrosis and apoptosis [87] [105]. Control and experimental cell populations were divided in a number of equal parts to analyze the development of their state after a 5-day exposure to the indicated DC MFs. (a) Viability of uninfected cells vs. viability of infected cells; (b) Necrosis of uninfected cells vs. necrosis of infected cells; (c) Apoptosis of uninfected cells vs. apoptosis of infected cells. Open squares are infected macrophages; closed circles are uninfected macrophages. The values represent the mean of three experiments. The student's paired $t$-test showed p-values of $<0.004$ for (a), $<0.01$ for (b), and $<0.05$ for (c).

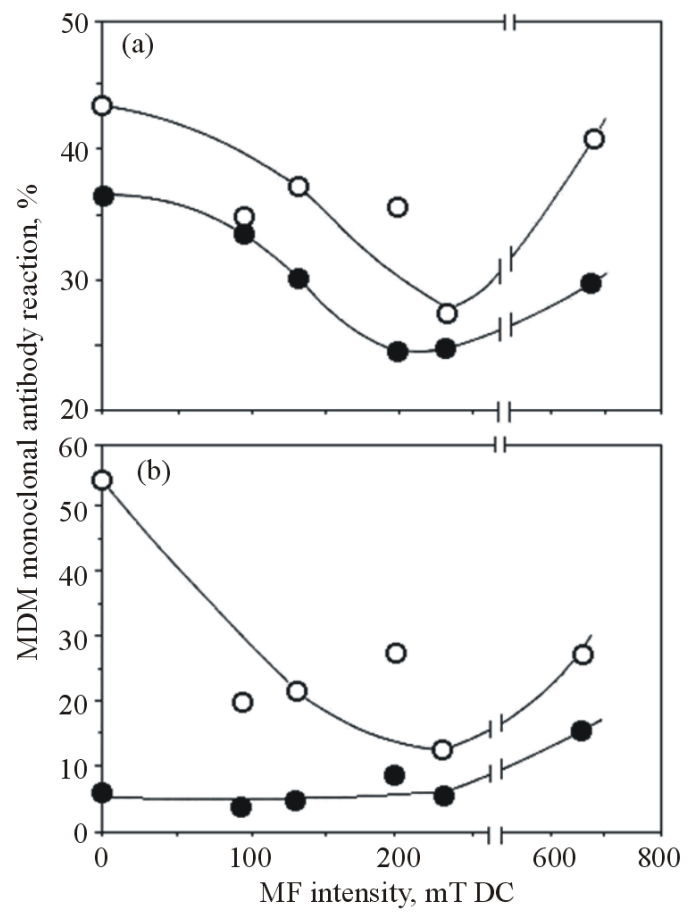

Figure 3. In human macrophages, the infection with Mycobacterium tuberculosis attenuated the MF- dependent variations of the CD14 and CD64 membrane protein expression [87] [105]. Control and experimental cell populations were divided in a number of equal parts to analyze, after a 5-day exposure to the indicated DC MFs, the labelling of CD14 (a) and CD64 (b) markers. Open circles are uninfected cell cultures; closed circles are infected cell cultures. The points represent the mean of three experiments. The student's paired t-test showed p-values of $<0.02$ for (a), and $<0.03$ for (b). 


\section{Acknowledgements}

The aid of all coworkers is greatly appreciated. A gratitude is particularly expressed to T. Eremenko for discussion pertaining the background effort of Biophysics to elucidate the mechanisms through which the magnetic fields influence the living matter.

\section{References}

[1] David, P. (1904) Sur la stabilité de la direction d'aimantation dans quelques roches volcaniques. Comptes Rendus de I'Acadamie des Science, Paris, 138, 41-42.

[2] Barnes, T.G. (1973) Origin and Destiny of the Earth’s Magnetic Field. I.C.R. Technical Monograph 4, Institute for Creation Research, San Diego.

[3] Jacobs, J.A. (1994) Reversals of the Earth’s Magnetic Field. University Press, Cambridge. http://dx.doi.org/10.1017/CBO9780511524929

[4] Merrill, R.T., McElhinny, M.W. and McFadden, P.L. (1998) The Magnetic Field of the Earth, Paleomagnetism, the Core, and the Deep Mantle. Academic Press, London.

[5] Goddard Geomagnetic Group (2004) Terrestrial Magnetism. NASA Space Flight Center, Greenbelt.

[6] Korhonen, J.V., Fairhead, J.D., Hamoudi, M., Hemant, K., Lesur, V., Mandea, M., Maus, S., Purucker, M., Ravat, D., Sazonova, T. and Thébault, E. (2007) Magnetic Anomaly Map of the World, Paris.

[7] Vladimirsky, B.M. (1980) Biological Rhythms and the Solar Activity. Problems of Cosmic Biology, 41, $289-315$.

[8] Meyer-Vernet, N. (2007) Basics of the Solar Wind. University Press, Cambridge. http://dx.doi.org/10.1017/CBO9780511535765

[9] Zhadin, M.N. (2001) Review of Russian Literature on Biological Action of DC and Low-Frequency AC Magnetic Fields. Bioelectromagnetics, 22, 27-45. http://dx.doi.org/10.1002/1521-186X(200101)22:1<27::AID-BEM4>3.0.CO;2-2

[10] Temuryants, N.A., Vladimirsky, B.M. and Tishkin, O.G. (1992) Extremely Low Frequency Electromagnetic Signals in the Biological World. Naukova Dumka, Kiev.

[11] Sentman, D.D. (1995) Schumann Resonances. In: Volland, H., Ed., Handbook of Atmospheric Electrodynamics, CRC Press, Boca Raton, 267-296.

[12] Alferov, O.A. and Kuznetsova, T.V. (1981) Effect of an Attenuated Geomagnetic Field on E. coli Tolerance to Ultraviolet Irradiation. Cosmiceskaja Biologhja i Aviacosmiceskaja Medizina, 15, 57-58.

[13] Bogatina, N.I., Litvin, V.M. and Travkin, M.P. (1986) Orientation of Wheat Roots on Exposure to a Geomagnetic Field. Biophysics, 31, 966-971.

[14] Belyavskaya, N.A., Fomicheva, V.M., Govorun, R.D. and Danilov, V.I. (1992) Structural-Functional Organisation of the Meristem Cells of Pea, Lentil and Flax Roots in Conditions of Screening the Geomagnetic Field. Biophysics, 37, 657-666.

[15] Fomicheva, V.M., Zaslavskii, V.A., Govorun, R.D. and Danilov, V.T. (1992) Dynamics of RNA and Protein Synthesis in the Cells of the Root Meristems of the Pea, Lentil and Flax. Biophysics, 37, 649-656.

[16] Govorun, R.D., Danilov, V.I., Fomicheva, V.M., Belyavskaya, N.A. and Zinchenko, S.Y. (1992) Influence of Fluctuation of the Geomagnetic Field and Its Screening on the Early Phases of the Development of Higher Plants. Biophysics, 37, 639-644.

[17] Sosunov, A.V., Golubchak, B.A., Semkin, V.Y. and Melnikov, A.V. (1972) Observation of Some Biological Processes in Shielded Spaces. In: Sanitary Assessment of Magnetic Fields, Academic Press, Moscow, 144-146.

[18] Kaznacheev, V., Mikhailova, V., Ivanova, M.P., Zaitsev, Y.A. and Kharina, N.I. (1989) Peculiarities of the Growth and Behavior of the Cell Monolayer in the Hypomagnetic Field. Problems of Cosmic Biology, 65, 189-195.

[19] Podkovkin, V.G. (1995) Response of Hormonal-Mediator Regulation Systems to the Weak Geomagnetic Fields Following Ionizing Radiation Effect. Radiation Biology and Radioecology, 35, 906-909.

[20] Grigoriev, Y.G. (1995) Reaction of Organism to Weakened Geomagnetic Field: The Effect of Magnetic Deprivation. Radiation Biology and Radioecology, 35, 3-18.

[21] Yeagley, H.L. and Whitmore, F.C. (1947) A Preliminary Study of Physical Basis of Bird Navigation. Journal of Applied Physics, 18, 1035-1063. http://dx.doi.org/10.1063/1.1697587

[22] Kholodov, Y.A. (1975) Reactions of the Nervous System to Electromagnetic Fields. Nauka, Moscow.

[23] Kirschvink, J.L., Jones, D.S. and MacFadden, B.J. (1985) Magnetite Biomineralization and Magnetoreception in Organisms: A New Biomagnetism. Plenum Press, New York. http://dx.doi.org/10.1007/978-1-4613-0313-8 
[24] Barnes, F.S. (1986) Interaction of DC and ELF Electric Fields with Biological Materials and Systems. In: Polk, C. and Postow, E., Eds., Handbook on Biological Effects of Electromagnetic Fields, CRC Press, Boca Raton, 103-147.

[25] Broun, G., Ilyinsky, O.B., Muraveiko, V., Gorshkov, E.S. and Pochtarev, V.I. (1977) Perception of Magnetic Field by Receptors of Lorenzini Ampullae in Black Sea Skates. Physiologhiceskij Zurnal SSSR, 63, 232-238.

[26] Ashkaliyev, Y.F., Drobzhev, V.I., Somsikov, V.M., Turkeyeva, V.A. and Yakovets, T.K. (1995) Effect of Heliogeophysical Parameters on the Ecological Situation. Biophysics, 40, 1035-1041.

[27] Villoresi, G., Breus, T.K., Dorman, L.I., Iucci, N. and Rapoport, S.I. (1995) Effect of Interplanetary and Geomagnetic Disturbances on the Rise in the Number of Clinically Severe Medical Pathologies (Myocardial Infarction and Stroke). Biophysics, 40, 983-993.

[28] Oraevskii, V., Golyshev, S., Levitin, A., Breus, T., Ivanova, S., Komarov, F. and Rapoport, S. (1995) Parameters of "Electromagnetic Weather" in Near-Earth Space Determining the Degree of Its Biotropicity. Biophysics, 40, 799-807.

[29] Sidyakin, V., Temuryants, N.A., Melnichenko, E.V. and Korenyuk, I.I. (1989) Relationship between Geomagnetic Disturbances and Evoked Activity in Neurons of the Motor Cortex. Problems of Cosmic Biology, 65, 81-87.

[30] Chibisov, S.M., Breus, T.K., Levitin, A.Y. and Drogova, G.M. (1995) Biological Effects of a Planetary Magnetic Storm. Biophysics, 40, 957-966.

[31] Belisheva, N.K. and Popov, A.N. (1995) Dynamics of the Morphofunctional State of Cell Cultures with Variation in the Geomagnetic Field in High Latitudes. Biophysics, 40, 737-745.

[32] Gmitrov, J. and Ohkubo, C. (2002) Artificial Static and Geomagnetic Field Interrelated Impact on Cardiovascular Regulation. Bioelectromagnetics, 23, 329-338. http://dx.doi.org/10.1002/bem.10020

[33] Gmitrov, J., Ohkubo, C. and Okano, H. (2002) Effect of 0.25 T Static Magnetic Field on Microcirculation in Rabbits. Bioelectromagnetics, 23, 224-229. http://dx.doi.org/10.1002/bem.10007

[34] Chiabrera, A., Bianco, B., Kaufman, J.J. and Pilla, A.A. (1991) Quantum Dynamics of Ions in Molecular Crevices under Electromagnetic Exposure. In: Brighton, C.T. and Pollak, S.R., Eds., Electromagnetics in Biology and Medicine, San Francisco Press, San Francisco, 21-26.

[35] Liboff, A.R. (1985) Geomagnetic Cyclotron Resonance in Living Cells. Journal of Biological Physics, 13, 99-102. http://dx.doi.org/10.1007/BF01878387

[36] Blanchard, J.P. and Blackman, C.F. (1994) Clarification and Application of an Ion Parametric Resonance Model for Magnetic Field Interactions with Biological Systems. Bioelectromagnetics, 15, 217-238. http://dx.doi.org/10.1002/bem.2250150306

[37] Volobuyev, A.N., Zhukov, B.N., Bakhito, A.U., Ovchinnikov, Y.L. and Trufanov, L.A. (1993) Influence of the Constant Magnetic Field and Laser Radiation on Neurophysiological Processes. Biophysics, 38, 371-376.

[38] Agulova, A.P., Opalinskaya, A.M. and Kiryanov, V.C. (1989) Typical Features of Reactions of Objects of Various Nature Sensitive to Change in Cosmophysical Factors and Action of Weak Artificial Electromagnetic Fields. Problems of Cosmic Biology, 65, 160-181.

[39] Piccardi, G. (1962) The Chemical Basis of Medical Climatology. Thomas, Springfield.

[40] Kislovsky, L.D. (1982) Reaction of Biological System to Weak Low-Frequency Electromagnetic Fields Adequate for It. Problems of Cosmic Biology, 43, 148-166.

[41] Zhvirblis, V.E. (1989) On Reproducibility of Heliobiological Experiments. Problems of Cosmic Biology, 65, 145-160.

[42] Lednev, V.V. (1991) Possible Mechanism for the Influence of Weak Magnetic Fields on Biological Systems. Bioelectromagnetics, 12, 71-75. http://dx.doi.org/10.1002/bem.2250120202

[43] Lednev, V.V., Srebnitskaya, L., Rozhdestvenskaya, Z., Klimov, A., Belova, N. and Tiras, K. (1996) Magnetic Parametric Resonance in Biosystems: Experimental Verification of the Predictions of a Theory Using Regenerating Planarians Dugesia tigrina as a Test System. Biophysics, 41, 825-836.

[44] Binghi, V.N. (1997) The Mechanism of Magnetosensitive Binding of Ions by Some Proteins. Biophysics, 42, $317-322$.

[45] Binghi, V.N. (2002) Magnetobiology: Underlying Physical Problems. Academic Press, San Diego.

[46] Emura, R., Ashida, N., Higashi, T. and Takeuchi, T. (2001) Orientation of Bull Sperms in Static Magnetic Fields. Bioelectromagnetics, 22, 60-65. http://dx.doi.org/10.1002/1521-186X(200101)22:1<60::AID-BEM7>3.0.CO;2-A

[47] El-Lakkani, A. (2001) Dielectric Response of Some Biological Tissues. Bioelectromagnetics, 22, 272-279. http://dx.doi.org/10.1002/bem.50

[48] Edmonds, E.D. (1993) Larmor Precession as a Mechanism for the Detection of Static and Alternating Magnetic Fields. Bioelectrochemistry and Bioenergetics, 30, 3-12. http://dx.doi.org/10.1016/0302-4598(93)80057-2

[49] Zhadin, M.N. (1996) Effect of Magnetic Fields on the Motion of an Ion in a Macromolecule: Theoretical Analysis. Biophysics, 41, 843-860. 
[50] Binghi, V.N. (2007) A Few Remarks on "Combined Action of DC and AC Magnetic Fields on Ion Motion in a Macromolecule”. Bioelectromagnetics, 28, 409-412. http://dx.doi.org/10.1002/bem.20314

[51] Zhadin, M.N. and Barnes, F. (2005) Frequency and Amplitude Windows in the Combined Action of DC and Low Frequency AC MFs on Ion Thermal Motion in a Macromolecule: Theoretical Analysis. Bioelectromagnetics, 26, 323-330. http://dx.doi.org/10.1002/bem.20095

[52] Adair, R.K. (2006) Comment: Analyses of Models of Ion Actions under the Combined Action of AC and DC Magnetic Fields. Bioelectromagnetics, 27, 332-334. http://dx.doi.org/10.1002/bem.20204

[53] Blank, M. and Goodman, R. (1997) Do Electromagnetic Fields Interact Directly with DNA? Bioelectromagnetics, 18, 111-115. http://dx.doi.org/10.1002/(SICI)1521-186X(1998)19:2<136::AID-BEM14>3.0.CO;2-O

[54] Adair, R.K. (1998) Extremely Low Frequency Electromagnetic Fields Do Not Interact Directly with DNA. Bioelectromagnetics, 19, 136-137. http://dx.doi.org/10.1002/(SICI)1521-186X(1998)19:2<136::AID-BEM14>3.0.CO;2-O

[55] Del Moral, A., Azanza, M.J. and Perez-Bruzon, R.N. (2006) Models of Neurone Dynamics: Spontaneous and under ELF Alternating Magnetic Field. In: Kostarakis, P., Ed., Biological Effects of EMFs, University of Ioannina and NCSR Demokritos Publishers, Crete, 594-603.

[56] Bawin, S.M. and Adey, W.R. (1976) Sensitivity of Calcium Binding in Cerebral Tissue to Weak Environmental Electric Fields Oscillating at Low Frequency. Proceedings of the National Academy of Sciences of the United States of America, 73, 1999-2003. http://dx.doi.org/10.1073/pnas.73.6.1999

[57] Bistolfi, F. (1991) Biostructures and Radiation Order Disorder. Minerva Medica, Torino.

[58] Ayrapetyan, S.N., Grigorian, K.V., Avanesian, A. and Stamboltsian, K.V. (1994) Magnetic Fields Alter Electrical Properties of Solutions and Their Physiological Effects. Bioelectromagnetics, 15, 133-142. http://dx.doi.org/10.1002/bem.2250150205

[59] Domrachev, G.A., Rodygin, Y.L. and Selivanovskii, D.A. (1992) The Role of Sound and of Liquid Water as a Dynamically Unstable Polymeric System in Mechanochemically Activated Oxygen-Generating Processes under Terrestrial Conditions. Russian Journal of Physical Chemistry, 66, 457-460.

[60] Giorgio, M., Trinei, M., Migliaccio, E. and Pelicci, P.G. (2007) Hydrogen Peroxide: A Metabolic By-Product or a Common Mediator of Ageing Signals? Nature Reviews: Molecular and Cell Biology, 8, 722-728. http://dx.doi.org/10.1038/nrm2240

[61] Hunanyan, A.S. and Ayrapetyan, S.N. (2007) Effect of Hydrogen Peroxide on Neuromembrane Chemosensitivity. Electromagnetic Biology and Medicine, 26, 225-233. http://dx.doi.org/10.1080/15368370701583164

[62] Adey, W.R. (1993) Biological Effects of Electromagnetic Fields. Journal of Cell Biochemistry, 51, 410-416. http://dx.doi.org/10.1002/jcb.2400510405

[63] Kaczmarek, L.K. (2007) Non-Conducting Functions of Voltage-Gated Ion Channels. Nature Reviews: Neuroscinces, 7, 761-771. http://dx.doi.org/10.1038/nrn1988

[64] Buchachenko, A.L. and Frankevich, E.L. (1993) Chemical Generation and Reception of Radio- and Microwaves. VCH Publishers, New York.

[65] Buchachenko, A.L., Kuznetsov, D.A. and Berdinsky, V.L. (2006) New Mechanisms of Biological Effects of Electromagnetic Fields. Biophysics, 51, 489-496. http://dx.doi.org/10.1134/S0006350906030249

[66] Buchachenko, A.L. and Berdinsky, V.L. (2002) Electron Spin Catalysis. Chemical Review, 102, 603-612. http://dx.doi.org/10.1021/cr010370l

[67] Lawrence, A.F. and Adey, W.R. (1982) Nonlinear Wave Mechanisms in Interactions between Excitable Tissues and Electromagnetic Fields. Neurological Research, 4, 115-153.

[68] Basset, C.A.L. (1993) Beneficial Effects of Electromagnetic Fields. Journal of Cell Biochemistry, 51, 387-393. http://dx.doi.org/10.1002/jcb.2400510402

[69] Walleczek, J. (1992) Electromagnetic Field Effects on Cells of the Immune System: The Role of Calcium Signaling. FASEB Journal, 6, 3177-3185.

[70] Liburdy, R.P. (1992) Calcium Signaling in Lymphocytes and ELF Fields: Evidence for an Electric Field Metric and a Site of Interaction Involving the Calcium Ion Channel. FEBS Lettters, 301, 53-59. http://dx.doi.org/10.1016/0014-5793(92)80209-Y

[71] Shahidain, R., Mullins, R.D. and Sisken, J.E. (2001) Calcium Spiking Activity and Baseline Calcium Levels in ROS 17/2.8 Cells Exposed to Extremely Low Frequency Electromagnetic Fields (ELF EMF). International Journal of Radiation Biology, 77, 241-248. http://dx.doi.org/10.1080/09553000010013779

[72] Testylier, G., Tonduli, L., Malabiau, R. and Debouzy, J.C. (2002) Effects of Exposure to Low Level Radiofrequency Fields on Acetylcholine Release in Hippocampus of Freely Moving Rats. Bioelectromagnetics, 23, 249-255.

http://dx.doi.org/10.1002/bem.10008 
[73] Fiorani, M., Cantoni, O., Sestili, P., Conti, R., Nicolini, P., Vetrano, F. and Dachà, M. (1992) Electric and/or Magnetic Field Effects on DNA Structure and Function in Cultured Human Cells. Mutation Research Letters, 282, 25-29. http://dx.doi.org/10.1016/0165-7992(92)90069-T

[74] Repacholi, M., Basten, A., Gebski, V., Noonan, D., Finnie, J. and Harris, A. (1997) Lymphomas in E $\mu-P i m 1$ Transgenic Mice Exposed to Pulsed 900 MHz Electromagnetic Fields. Radiation Research, 147, 631-640. http://dx.doi.org/10.2307/3579630

[75] Ding, G.R., Wake, K., Taki, M. and Miyakoshi, J. (2001) Increase in Hypoxanthine-Guanine Phosphoribosyl Transferase Gene Mutations by Exposure to Electric Field. Life Sciences, 68, 1041-1046. http://dx.doi.org/10.1016/S0024-3205(00)01007-9

[76] Herada, S.I., Yamada, S., Kuramata, O., Gunji, Y., Kawasaki, M., Miyakawa, T., Yonekura, H., Sakurai, S., Bessho, K., Hosono, R. and Yamamoto, H. (2001) Effects of High ELF Magnetic Fields on Enzyme-Catalyzed DNA and RNA Synthesis in Vitro and on a Cell-Free DNA Mismatch Repair. Bioelectromagnetics, 22, 260-268. http://dx.doi.org/10.1002/bem.48

[77] Nindl, G., Hughes, E., Johnson, M., Spandau, D., Vesper, D. and Balcavage, W.X. (2002) Effect of Ultraviolet B Radiation and $100 \mathrm{~Hz}$ Electromagnetic Fields on Proliferation and DNA Synthesis of Jurkat Cells. Bioelectromagnetics, 23, 455-463. http://dx.doi.org/10.1002/bem.10039

[78] Ravera, S., Repaci, E., Morelli, A., Pepe, I.M., Botter, R. and Beruto, D. (2004) Electromagnetic Field of Extremely Low Frequency Decreased Adenylate Kinase Activity in Retinal Rod Outer Segment Membranes. Bioelectrochemistry, 63, 317-320. http://dx.doi.org/10.1016/j.bioelechem.2003.10.029

[79] Volpe, P. and Eremenko, T. (2005) Genome Stability vs. Deprivation or Enrichment of the Geomagnetic Field. Environmentalist, 25, 72-82. http://dx.doi.org/10.1007/s10669-005-4269-7

[80] Volpe, P. and Eremenko, T. (2005) Gene Expression in a Space-Simulating Magnetically Shielded Environment. Environmentalist, 25, 83-92. http://dx.doi.org/10.1007/s10669-005-4270-1

[81] Volpe, P., Parasassi, T., Esposito, C., Ravagnan, G., Giusti, A.M., Pasquarelli, A. and Eremenko, T. (1998) Cell Membrane Lipid Molecular Dynamics in a Solenoid versus a Magnetically Shielded Room. Bioelectromagnetics, 19, 107111. http://dx.doi.org/10.1002/(SICI)1521-186X(1998)19:2<107::AID-BEM8>3.0.CO;2-5

[82] Gölfert, F., Hofer, A., Thümmler, M., Bauer, H. and Funk, R.H.W. (2001) Extremely Low Frequency Electromagnetic Fields and Heat Shock Can Increase Microvesicle Motility in Astrocytes. Bioelectromagnetics, 22, 71-78. http://dx.doi.org/10.1002/1521-186X(200102)22:2<71::AID-BEM1008>3.0.CO;2-I

[83] Lino, M. and Okuda, Y. (2001) Osmolality Dependence of Erythrocyte Sedimentation and Aggregation in a Strong Magnetic Field. Bioelectromagnetics, 22, 46-52.

http://dx.doi.org/10.1002/1521-186X(200101)22:1<46::AID-BEM5>3.0.CO;2-0

[84] Eremenko, T., Esposito, C., Pasquarelli, A., Pasquali, E. and Volpe, P. (1997) Cell-Cycle Kinetics of Friend Erythroleukemia Cells in a Magnetically Shielded Room and in a Low-Frequency/Low-Intensity Magnetic Field. Bioelectromagnetics, 18, 58-66. http://dx.doi.org/10.1002/(SICI)1521-186X(1997)18:1<58::AID-BEM9>3.0.CO;2-4

[85] Cridland, N.A., Haylock, R.G.E. and Saunders, R.D. (1999) 50 Hz Magnetic Field Exposure Alters Onset of S-Phase in Normal Human Fibroblasts. Bioelectromagnetics, 20, 446-452. http://dx.doi.org/10.1002/(SICI)1521-186X(199910)20:7<446::AID-BEM6>3.0.CO;2-C

[86] Markkanen, A., Juutilainen, J., Lang, S., Pelkonen, J., Rytömaa, T. and Naarala, J. (2001) Effects of 50 Hz Magnetic Field on Cell Cycle Kinetics and the Colony Forming Ability of Budding Yeast Exposed to Ultraviolet Radiation. Bioelectromagnetics, 22, 345-350. http://dx.doi.org/10.1002/bem.60

[87] Volpe, P., Cappelli, G., Mariani, F., Serafino, A. and Eremenko, T. (2002) Macrophage Sensitivity to Static Magnetic Fields. In: Kostarakis, P., Ed., Biological Effects of EMFs, University of Ioannina and NCSR Demokritos, Rhodes, 374-381.

[88] Volpe, P. (2003) Interactions of Zero-Frequency and Oscillating Magnetic Fields with Biostructures and Biosystems. Photochemical and Photobiological Sciences, 2, 637-648. http://dx.doi.org/10.1039/b212636b

[89] Obo, M., Konishi, S., Otaka, Y. and Kitamura, S. (2002) Effect of Magnetic Field Exposure on Calcium Channel Currents Using Patch Clamp Technique. Bioelectromagnetics, 23, 306-314. http://dx.doi.org/10.1002/bem.10022

[90] Wang, X., Becker, F. and Gascoyne, P.R.C. (2002) Membrane Dielectric Changes Indicate Induced Apoptosis in HL60 Cells More Sensitively than Surface Phosphatidylserine Expression or DNA Fragmentation. Biochimica et Biophysica Acta, Biomembranes, 1564, 412-420. http://dx.doi.org/10.1016/S0005-2736(02)00495-9

[91] Gidon-Jeangirard, C., Solito, E., Hofmann, A., Russo-Marie, F., Freyssinet, J.M. and Martinez, M.C. (1999) Annexin V Counteracts Apoptosis While Inducing $\mathrm{Ca}^{2+}$ Influx in Human Lymphocytic T Cells. Biochemical and Biophysical Research Communications, 265, 709-715. http://dx.doi.org/10.1006/bbrc.1999.1752

[92] Fanelli, C., Coppola, S., Barone, R., Colussi, C., Gualardi, G., Volpe, P. and Ghibelli, L. (1999) Magnetic Fields In- 
crease Cell Survival by Inhibiting Apoptosis via Modulation of $\mathrm{Ca}^{2+}$ Influx. FASEB Journal, 13, 95-102.

[93] Delgado, J.M.R., Leal, J., Moneagudo, J.L. and Garcia, M.G. (1982) Embryological Changes Induced by Weak, Extremely Low Frequency Electromagnetic Fields. Journal of Anatomy, 134, 533-551.

[94] Grimaldi, S., Pozzi, D., Lisi, A., Rieti, S., Manni, V., Ravagnan, G., Giuliani, L., Eremenko, T. and Volpe, P. (2000) Influence of the Magnetic Field on the Tadpole Metamorphosis. International Journal of Radiation Medicine, 1, 96103.

[95] Mirabolghasemi, G. and Azarnia, M. (2002) Developmental Changes in Drosophila melanogaster Following Exposure to Alternating Electromagnetic Fields. Bioelectromagnetics, 23, 416-420. http://dx.doi.org/10.1002/bem.10042

[96] Markov, M.S. (2007) Magnetic Field Therapy: A Review. Electromagnetic Biology and Medicine, 26, 1-23. http://dx.doi.org/10.1080/15368370600925342

[97] McCann, J., Dietrich, F., Rafferty, C. and Martin, A.O. (1993) A Critical Review of the Genotoxic Potential of Electric and Magnetic Fields. Mutation Researches, 297, 61-95. http://dx.doi.org/10.1016/0165-1110(93)90008-B

[98] Liburdy, R.P., Sloma, T.R., Sokolic, R. and Yaswen, P. (1993) ELF Magnetic Fields, Breast Cancer, and Melatonin: 60 $\mathrm{Hz}$ Fields Block Melatonin's Oncostatic Action on ER ${ }^{+}$Breast Cancer Cell Proliferation. Journal of Pineal Research, 14, 89-97. http://dx.doi.org/10.1111/j.1600-079X.1993.tb00491.x

[99] Nordenson, I., Mild, K.H., Andersson, G. and Sandström, M. (1994) Chromosomal Aberrations in Human Amniotic Cells after Intermittent Exposure to Fifty Hertz Magnetic Fields. Bioelectromagnetics, 15, 293-301. http://dx.doi.org/10.1002/bem.2250150404

[100] Miyakoshi, J., Yamagishi, N., Ohtsu, S., Mohri, K. and Takebe, H. (1996) Increase in Hypoxanthine-Guanine Phosphoribosyl Transferase Gene Mutations by Exposure to High-Density 50-Hz Magnetic Fields. Mutation Researches/ Fundamental and Molecular Mechanisms of Mutagenesis, 349, 109-114. http://dx.doi.org/10.1016/0027-5107(95)00166-2

[101] Morandi, M.A., Pak, C.M., Caren, R.P. and Caren, L.D. (1996) Lack of an EMF-Induced Genotoxic Effect in the Ames Assay. Life Sciences, 59, 263-271. http://dx.doi.org/10.1016/0024-3205(96)00291-3

[102] Lacy-Hulbert, A., Metcalfe, J.C. and Hesketh, R. (1998) Biological Responses to Magnetic Fields. FASEB Journal, 12, 395-420.

[103] Cantoni, O., Sestili, P., Fiorani, M. and Dachà, M. (1996) Effect of 50 Hz Sinusoidal Electric and/or Magnetic Fields on the Rate of Repair of DNA Single Stand Breaks in Cultured Mammalian Cells Exposed to Three Different Carcinogens: Methylmethane Sulphonate, Chromate and 254 nm UV Radiation. Biochemistry and Molecular Biology International, 38, 527-533.

[104] Zhang, H., Yang, X., Li, Z., Zeng, F., Liu, H., Deng, R. and Du, B. (1999) Inducing Apoptosis of Cancer Cell and Inhibiting Mice’s Malignant Tumour Growth by Magnetic Fields. Wuhan University Journal of Natural Sciences, 4, 363366. http://dx.doi.org/10.1007/BF02842374

[105] Mariani, F., Cappelli, G., Eremenko, T. and Volpe, P. (2001) Influence of Static Magnetic Fields on Cell Viability, Necrosis and Apoptosis. Journal of Biological Research, 77, 71-84.

[106] Buemi, M., Marino, D., Di Pasquale, G., Floccari, F., Senatore, M., Aloisi, C., Grasso, F., Mondio, G., Perillo, P., Frisina, N. and Corica, F. (2001) Cell Proliferation/Cell Death Balance in Renal Cell Cultures after Exposure to a Static Magnetic Field. Nephron, 87, 269-273. http://dx.doi.org/10.1080/09553000400029502

[107] Santini, M.T., Ferrante, A., Rainaldi, G., Indovina, P. and Indovina, P.L. (2005) Extremely Low Frequency (ELF) Magnetic Fields and Apoptosis: A Review. International Journal of Radiation Biology, 81, 1-11. http://dx.doi.org/10.1080/09553000400029502

[108] Valiron, O., Peris, L., Rikken, G., Schweitzer, A., Saoudi, Y., Remy, C. and Job, D. (2005) Cellular Disorders Induced by High Magnetic Fields. Journal of Magnetic Resonance Imaging, 22, 334-340. http://dx.doi.org/10.1002/jmri.20398

[109] Potenza, L., Cucchiarini, L., Vetrano, F. and Dachà, M. (2005) Electric and Magnetic Fields as Possible Risk Factors for Human Health. International Journal of Risk Assessment and Management, 5, 292-310. http://dx.doi.org/10.1504/IJRAM.2005.007173

[110] Volpe, P. and Eremenko, T. (2007) Mechanisms of the Target Response to Magnetic Fields and Their Correlation with the Biological Complexity. The Environmentalist, 27, 387-393. http://dx.doi.org/10.1007/s10669-007-9085-9

[111] Kuzin, A.M. (1970) Problemi Radiobiologhii. Izdatelstvo Znanje, Moskwa, 19-26.

[112] Markov, M.S. (2004) Myosin Phosphorylation: A Plausible Tool for Studying Biological Windows. In: Kostarakis, P., Ed., Biological Effects of EMFs, University of Ioannina and NCSR Demokritos, Kos, 1-9.

[113] Timoféeff-Ressovsky, N. and Zimmer, K. (1947) Biophysik, Bd. 1: Das Trefferprinzip in der Biologie, Hirzel, Leipzig.

[114] Elkind, M., Sutton, H. and Moses, W. (1961) Postirradiation Survival Kinetics of Mammalian Cells Grown in Culture. Journal of Cellular and Comparative Physiology, 58, 113-134. http://dx.doi.org/10.1002/jcp.1030580412 
Scientific Research Publishing (SCIRP) is one of the largest Open Access journal publishers. It is currently publishing more than 200 open access, online, peer-reviewed journals covering a wide range of academic disciplines. SCIRP serves the worldwide academic communities and contributes to the progress and application of science with its publication.

Other selected journals from SCIRP are listed as below. Submit your manuscript to us via either submit@scirp.org or Online Submission Portal.
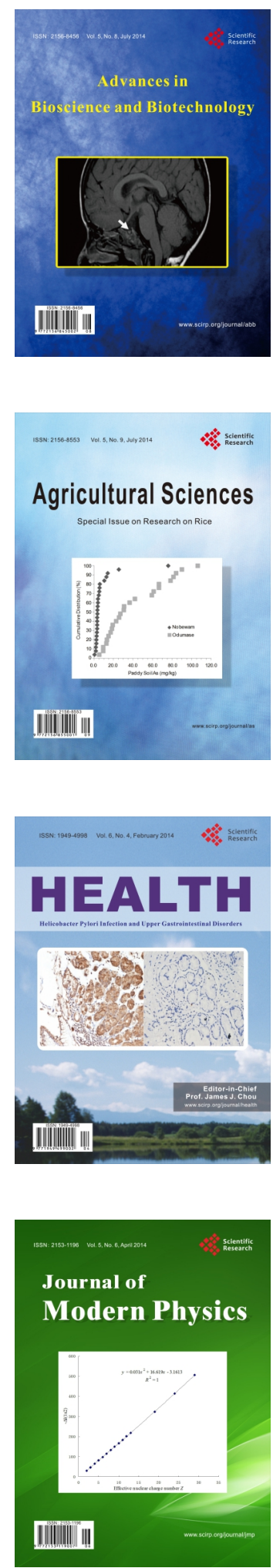
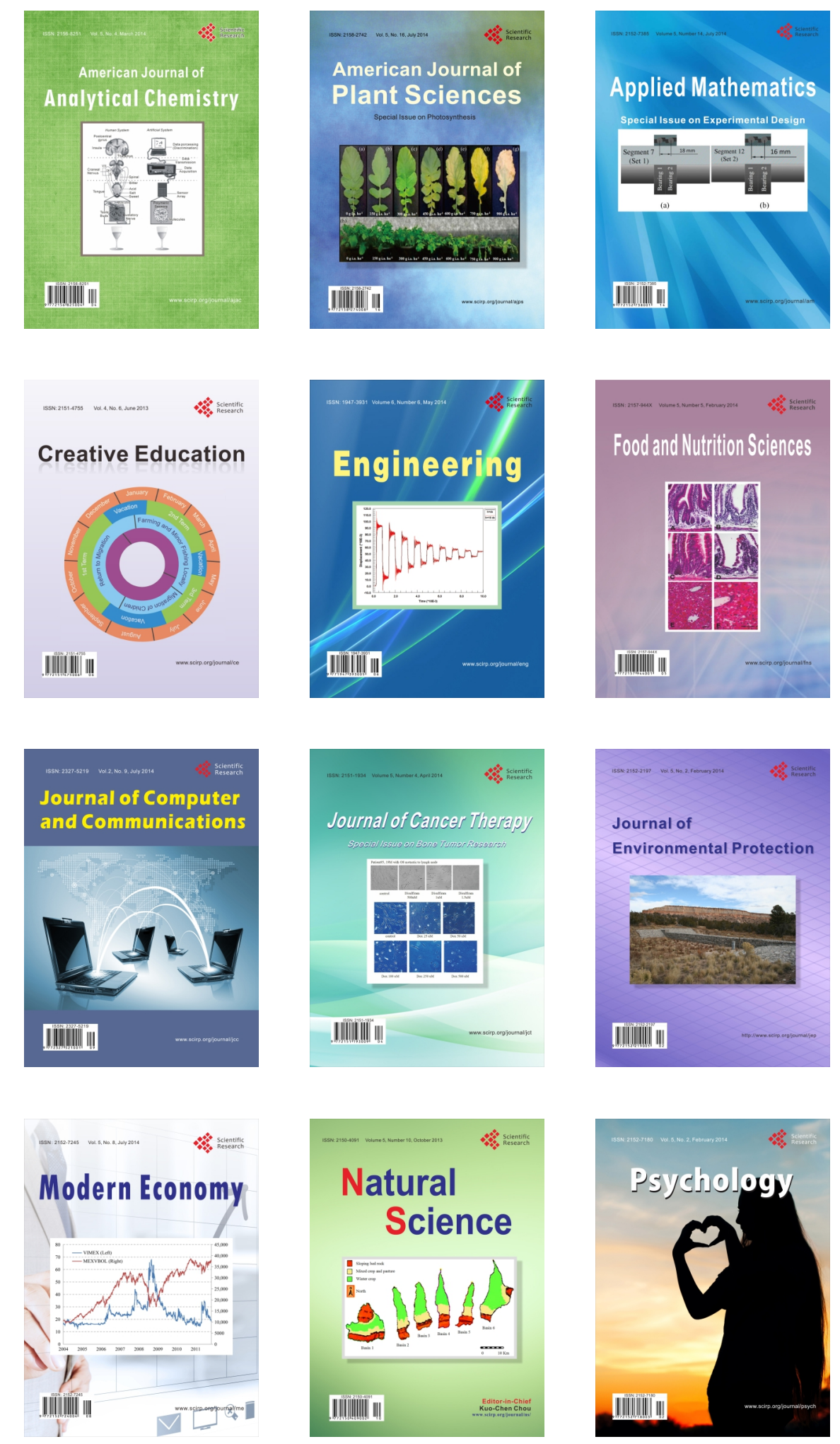